\title{
Lung cancer: the importance of seeing a respiratory physician
}

\author{
R.J. Fergusson*, C.S. Thomson \#, , D.H. Brewster" , P.H. Brown ${ }^{+}$, R. Milroy ${ }^{\S}$, on behalf of the Scottish \\ Cancer Trials Lung Group and the Scottish Cancer Therapy Network
}

Lung cancer: the importance of seeing a respiratory physician. R.J. Fergusson, C.S. Thomson, D.H. Brewster, P.H. Brown, R. Milroy, on behalf of the Scottish Cancer Trials Lung Group and the Scottish Cancer Therapy Network. (C)ERS Journals Ltd 2003.

ABSTRACT: Patients with lung cancer present to and are managed by a variety of clinicians. In this study the effect of involvement by a respiratory physician on the diagnosis, staging, treatment and survival of a large unselected group of lung cancer patients was investigated.

The study population was derived from the Scottish Cancer Registry. A total of 3,855 patients diagnosed during 1995 with lung cancer were studied. The data were validated and supplemented by references to medical records.

The study found that a respiratory physician had been involved in the initial management of $2,901(75.3 \%)$ patients. These patients were found more likely to have had the cancer diagnosis confirmed by histological methods and to have received active treatment with surgery, radiotherapy or chemotherapy. Survival, 1 yr after diagnosis was higher in patients who saw a respiratory physician $(24.4$ versus $11.1 \%)$ and benefit was found to have remained 3 yrs after diagnosis (8.1 versus $3.7 \%)$. Although the patients who had not seen a respiratory physician were generally older, and had more extensive disease, after correcting for age, stage and other prognostic factors, the relative hazard ratio of death for those not managed by a respiratory physician was 1.44.

The data from this study supports the recommendations of recent lung cancer guidelines for the early involvement by a respiratory physician.

Eur Respir J 2003; 21: 606-610.
*Western General Hospital, Lothian University Hospitals NHS Trust and \#Scottish Cancer Intelligence Unit, ISD Edinburgh and "Trent Cancer Registry, Weston Park Hospital, Sheffield and ${ }^{+}$Tayside University Hospitals Trust, Perth Royal Infirmary, Perth, \$Stobhill Hospital, and North Glasgow University Hospitals NHS Trust, Glasgow, UK.

Correspondence: R FergussonWestern General Hospital, Lothian University Hospitals NHS Trust, Edinburgh, EH4 2XU, UK.

Fax: 441313433989

E-mail: ron.fergusson@luht.scot.nhs.uk

Keywords: Lung cancer, respiratory physician, survival, treatment

Received: July 92002

Accepted after revision: July 112002

The Scottish Cancer Therapy Network is funded by grants from the Clinical Resource and Audit Group (CRAG) and the Chief Scientist Office (CSO), both of the Scottish Executive Health Department.
Patients with lung cancer present to hospital services at the time of diagnosis in a number of ways [1]. Although many patients are referred by their general practioner (GP) to a respiratory physician (RP), a significant proportion are diagnosed and managed by other hospital clinicians (general physicians, geriatricians, accident and emergency etc.). Recent national guidelines for the management of lung cancer [2-4] stress the importance of referral to an RP at the time of diagnosis although there is little published evidence to support this recommendation. In an editorial on lung cancer management MUERS and HAWARD [5], stated that patients in Yorkshire who see an RP are more likely to get a definitive histological diagnosis and receive active treatment than those who do not. No details of case mix or outcome for the two groups were available.

A national, population-based study of the management and survival of lung cancer patients in Scotland has been conducted recently by the authors and a general overview of the results has been presented elsewhere [6]. In the present paper the authors report specifically on the role of the RP in the management of lung cancer. The characteristics of patients initially managed by an RP were examined along with whether this group of patients were more or less likely to have a microscopically confirmed diagnosis and if their stage of disease was recorded. The likelihood of whether patients seeing an RP are more likely to receive active treatment, and whether this translates into improved survival was also investigated.

$\overline{\text { For editorial comments see page } 565 .}$

\section{Methods}

\section{Patients}

The study was part of a comprehensive survey of the care patterns in patients diagnosed with lung cancer in Scotland in 1995 [6]. All the cases studied were identified from the Scottish Cancer Registry. The Directors of Public Health of each Health Board, Medical Directors of all National Health Service (NHS) Trusts and relevant consultants were contacted to obtain permission to examine case notes and all agreed. Information was abstracted from the medical records by specially trained Scottish Cancer Therapy Network (SCTN) data managers. Details concerning the consultants in charge at each stage of care from initial referral up to 6 months from diagnosis were collected. The specialties of consultants were assigned using the Manpower database held at the Information \& Statistics Division of the NHS in Scotland, supplemented by the British Thoracic Society Directory of Respiratory Medicine Services to ensure correct classification of respiratory and general physicians. Patients were classified according to whether they had or had not been managed by (referred by, diagnosed by or referred to) an RP within their first 6 months of care. Those patients with no contact with an $\mathrm{RP}$ in this period are known in this study as the "no RP involvement" or "non-RP" group. "Lung cancer specialists" are defined here as respiratory physicians, cardiothoracic surgeons or oncologists.

Information relating to clinical presentation, diagnostic 
and staging methods, clinical extent of disease, pathology and treatment received within 6 months of diagnosis was taken from the case notes. Simple classifications for the clinical extent of disease (local, regional, distant or unknown) were used in this analysis, therefore, few patients in Scotland were formally staged using the tumour nodes metastasis system. Morphology of the tumour was based on what was recorded on pathology reports. The treatment modalities examined were surgical resection, chemotherapy, and radiotherapy, received within 6 months of diagnosis. The term "any active treatment" was used to include any of these three therapies. The intent of treatment was poorly recorded and therefore not examined. The date of diagnosis was defined, in order of priority as one of the following: date of histological (excluding autopsy), cytological confirmation; diagnostic radiology; or diagnostic clinical examination. Demographic data included age, sex, social deprivation [7] and settlement size of place of residence. In Scotland the most commonly used measure of socioeconomic status for health data is the Carstairs index of deprivation, which is an area-based measure. It is derived from four variables (car ownership, heads of household in social class IV or V, overcrowding in the household and male unemployment) recorded in the 1991 Census. Both deprivation and settlement size were obtained for each postcode from the General Register Office for Scotland (GROS) and were matched to patients according to their postcode of residence. Survival data were obtained by linkage [8] to the GROS death records up to the end of 1998.

\section{Statistical analysis}

Chi-squared tests were used to examine whether there were differences in the proportion of patients managed by an RP for the various demographic factors. Similarly, Chi-squared tests of association were performed to examine if there were any differences in the distribution of the clinical extent of disease, use of staging and diagnostic investigations, microscopic verification and prescription of the different treatment modalities for patients managed by an RP and those patients with no RP involvement.

Survival curves and point estimates at 1 and 3 yrs for both groups were obtained using Kaplan-Meier analysis. Cox's proportional hazards modelling [9] was used to examine the effect of introducing other variables into the model on the hazard ratio of death of patients with no RP involvement relative to the group of patients managed by an RP.

\section{Results}

A total of 4,465 lung cancer patients were recorded on the Scottish Cancer Registry in 1995, of which 4,225 were found to be potentially eligible for analysis and of these, case notes could be located for 3,855 (91.2\%) patients. Further details of the ineligibility criteria are presented elsewhere [6]. From the total of 3,855 patients studied, 2,901 (75.3\%) were managed by a respiratory physician during the first 6 months of their care. The remaining 954 patients had no contact with an RP in this period.

Table 1 shows the proportion of cases in each of the demographic factors: age, sex, deprivation quintile and settlement size, being managed by an RP during the first 6 months of care. Younger patients ( $<70 \mathrm{yrs})$ were more likely to be seen by an RP than older patients $(\mathrm{p}<0.001)$. Males were more likely to see an RP than females ( $\mathrm{p}=0.04)$, and patients resident in rural areas were more likely to be managed by an RP than those resident in densely urban areas $(\mathrm{p}=0.03)$. Deprivation index, however, was similar in the two groups $(\mathrm{p}=0.18)$.
Table 1.-The number and percentages of patients with a respiratory physician (RP) involvement for each of the demographic factors

\begin{tabular}{|c|c|c|c|c|}
\hline & \multirow{2}{*}{$\frac{\text { All cases }}{\mathrm{n}}$} & \multicolumn{2}{|c|}{$\mathrm{RP}$ involvement } & \multirow[t]{2}{*}{ p-value ${ }^{\#}$} \\
\hline & & $\mathrm{n}$ & $\%$ & \\
\hline Age group yrs & & & & $<0.001$ \\
\hline$<0$ & 577 & 482 & 83.5 & \\
\hline $60-69$ & 1268 & 1013 & 79.9 & \\
\hline $70-79$ & 1444 & 1086 & 75.2 & \\
\hline $80+$ & 566 & 320 & 56.5 & \\
\hline Sex & & & & 0.04 \\
\hline Male & 2341 & 1789 & 76.4 & \\
\hline Female & 1514 & 1112 & 73.4 & \\
\hline Deprivation quintile & & & & 0.18 \\
\hline $1=$ least & 533 & 388 & 72.8 & \\
\hline 2 & 648 & 479 & 73.9 & \\
\hline 3 & 800 & 626 & 78.3 & \\
\hline 4 & 840 & 629 & 74.9 & \\
\hline $5=\operatorname{most}$ & 1034 & 779 & 75.3 & \\
\hline $\begin{array}{l}\text { Settlement size (number } \\
\text { of people in block) }\end{array}$ & & & & 0.03 \\
\hline$>100000$ & 1876 & 1378 & 73.5 & \\
\hline 500-99999 & 1758 & 1348 & 76.7 & \\
\hline Not in a locality (rural) & 220 & 174 & 79.1 & \\
\hline
\end{tabular}

\#. Chi-squared test for association; ${ }^{\top}$ : settlement size could not be assigned for one case.

\section{Management by a multidisciplinary lung cancer specialist team}

From the total of 2,901 patients managed by an RP within 6 months of diagnosis, $2,531(87.2 \%)$ were diagnosed by the RP. The 954 patients who had no RP involvement during their first 6 months of care were diagnosed by one of the following: general physician 357 (37.4\%); geriatrician 275 $(28.8 \%)$; cardiothoracic surgeon $81(8.5 \%)$, general surgeon $73(7.7 \%)$; and oncologists $13(1.4 \%)$, respectively. A total of $944(24.5 \%)$ of all the patients were only seen by an RP. Patients seeing an RP during the first 6 months of care were more likely to also see an oncologist $1,624(56.0 \%)$ versus 406 $(42.6 \%)$ who were not seen by an RP $(\mathrm{p}<0.001)$. Also patients managed by an RP were more likely to see a cardiothoracic surgeon $587(20.2 \%)$ versus $152(15.9 \%), \mathrm{p}=0.003$ who were not managed by an RP (fig. 1). Therefore, multidisciplinary lung cancer specialist management occurred for $1,957(67.5 \%)$ of the patients managed by an RP, but only for $91(9.5 \%)$ of the patients who were not. There were $487(12.6 \%)$ patients who were not managed by a lung cancer specialist at all during their first 6 months of care.

\section{Characteristics of the tumour and staging investigations}

Patients managed by an RP were significantly less likely to have their clinical extent of disease undetermined $(9.0 \%)$ than those patients not managed by an RP $(17.1 \%, \mathrm{p}<0.001)$. However respiratory physicians saw proportionately fewer patients with metastatic disease (table 2). For those with known stage, patients with distant metastases at presentation comprised $29.9 \%$ of the RP workload compared with $52.3 \%$ of the patients with no RP involvement.

There were significant differences in some of the investigations by which RP patients and non-RP patients were staged. Patients seen by an RP were more likely to: undergo a computed tomography (CT) of the chest than patients who 


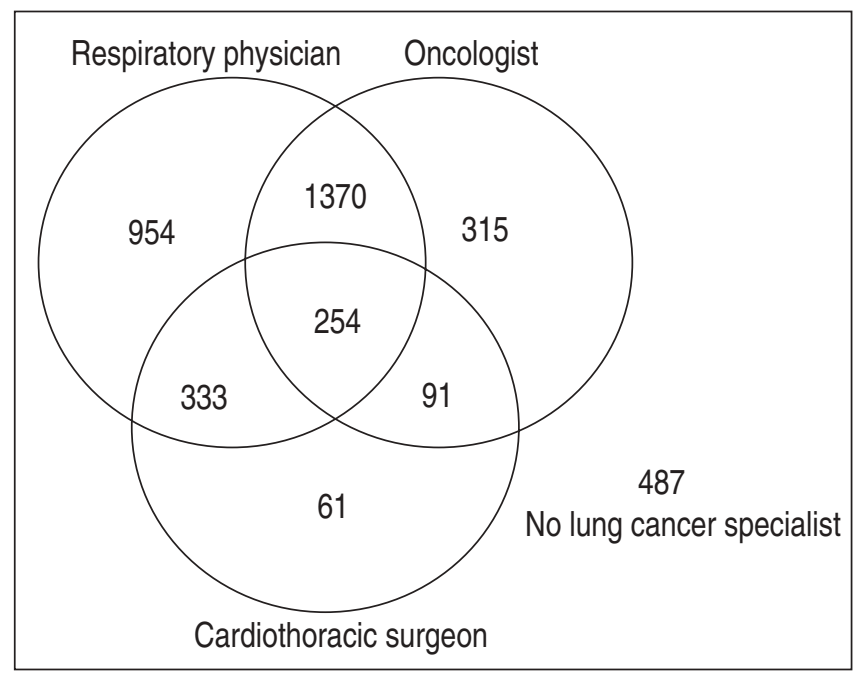

Fig. 1.-Number of patients managed by different lung cancer specialists involved in the care of patients with lung cancer.

were not seen by an RP (51.7 versus $32.7 \%, \mathrm{p}<0.001)$; receive more bone scans (19.1 versus $13.3 \% ; \mathrm{p}<0.001$ ); undergo more bronchoscopies (78.4 versus $32.9 \%, \mathrm{p}<0.001)$; and receive more mediastinoscopy staging $(8.2$ versus $4.9 \%, \mathrm{p}=0.001)$. In contrast, patients managed by an RP were less likely to have a liver ultrasound $(21.9$ versus $29.1 \%, \mathrm{p}<0.001)$ and also less likely to be staged with a CT scan of the brain (8.0 versus $14.5 \%, \mathrm{p}<0.001)$.

As well as having more thoroughly staged tumours, patients managed by an RP were more likely to have their tumours verified microscopically 2,307 patients $(79.5 \%)$ than the non-RP patients $549(57.5 \%, \mathrm{p}<0.001)$. Table 2 also shows the distribution of tumour morphologies excluding the 999 patients without microscopic verification. Although RPs appear to see more squamous cell carcinomas and fewer adenocarcinomas, these data may be skewed by the large differences between groups in microscopic verification.

\section{Treatments within 6 months}

Overall, RP patients were more likely to receive some form of active treatment than the non-RP group (63.2 versus $37.3 \%$, $\mathrm{p}<0.001$, table 3 ). More patients had their tumours resected when managed by an RP than those with no RP involvement (12.0 versus $6.7 \%, \mathrm{p}<0.001$ ). Of the 2,901 patients managed by
Table 3.-Distribution of treatment factors by a respiratory physician involvement or not

\begin{tabular}{|c|c|c|c|c|c|c|}
\hline \multirow[t]{2}{*}{ Treatment modality } & \multicolumn{2}{|c|}{$\mathrm{RP}$} & \multicolumn{2}{|c|}{ No RP } & \multicolumn{2}{|c|}{ All } \\
\hline & $\mathrm{n}$ & $\%$ & $\mathrm{n}$ & $\%$ & $\mathrm{n}$ & $\%$ \\
\hline \multicolumn{7}{|l|}{ Any active treatment ${ }^{\#}$} \\
\hline Given & 1832 & 63.2 & 356 & 37.3 & 2188 & 56.8 \\
\hline None & 1069 & 36.8 & 598 & 62.7 & 1667 & 43.2 \\
\hline \multicolumn{7}{|l|}{ Surgery } \\
\hline Resection & 347 & 12.0 & 64 & 6.7 & 411 & 10.7 \\
\hline Thoracotomy & 33 & 1.1 & 3 & 0.3 & 36 & 0.9 \\
\hline No surgery & 2521 & 86.9 & 887 & 93.0 & 3408 & 88.4 \\
\hline \multicolumn{7}{|l|}{ Radiotherapy $^{+}$} \\
\hline High dose chest ${ }^{\S}$ & 100 & 3.4 & 6 & 0.6 & 106 & 2.7 \\
\hline Low dose chest $f$ & 913 & 31.5 & 165 & 17.3 & 1078 & 28.0 \\
\hline Other sites & 123 & 4.2 & 70 & 7.3 & 193 & 5.0 \\
\hline No radiotherapy & 1762 & 60.7 & 712 & 74.6 & 2474 & 64.2 \\
\hline \multicolumn{7}{|l|}{ Chemotherapy**,开\# } \\
\hline SCLC & 367 & 69.0 & 58 & 39.7 & 425 & 62.7 \\
\hline Not SCLC ${ }^{++}$ & 142 & 8.3 & 28 & 7.6 & 170 & 8.2 \\
\hline
\end{tabular}

RP: respiratory physician; ${ }^{*}$ : any specific anti-cancer therapy (i.e. surgical resection, any chemotherapy or radiotherapy) within 6 months of diagnosis; ": thoracotomy only (not resectable); ${ }^{+}$: four cases with dose to chest unknown (three RP; one no RP) are not shown; ${ }^{\S}$ : dose to chest $\geqslant 50 \mathrm{~Gy} ;{ }^{f}$ : dose to chest $<50 \mathrm{~Gy}$; ${ }^{* *}$ : percentages are of cases with a particular cell type; ${ }^{\# \#}$ : 1,106 cases type unknown or not recorded, those with no microscopic verification are not shown, only 26 received some chemotherapy (23 RP; three no RP); ${ }^{++}$: includes 2,071 cases in total with a recorded histological type known not to be small cell carcinoma of the lung.

an RP, $1,139(39.3 \%)$ received some form of radiotherapy; and of these, the irradiation was to the chest for 1,016 $(89.2 \%)$. In comparison, only $242(25.4 \%)$ of the 954 patients with no RP involvement were given radiotherapy, and this was to the chest in only 172 cases $(71.1 \%)$. Both of these differences were highly significant $(p<0.001)$. Similarly, patients were more likely to be given chemotherapy if they were seen by an RP (18.3 versus $9.3 \%$ respectively; $\mathrm{p}<0.001)$. Of the 532 patients with small cell lung cancer (SCLC) who were seen by an RP, $69.0 \%$ received some form of chemotherapy compared to $39.7 \%$ of the 146 patients with SCLC not managed by an RP. When chemotherapy was given for SCLC, RP patients were more likely to receive combination rather than single-agent treatment than those not seeing an RP (82.0 versus $62.1 \%, \mathrm{p}<0.001)$.

Table 2.-Distribution of clinical extent of disease and tumour morphology by a respiratory physician (RP) involvement or not

\begin{tabular}{|c|c|c|c|c|c|c|}
\hline & \multicolumn{2}{|c|}{$\mathrm{RP}$ involvement } & \multicolumn{2}{|c|}{ No RP involvement } & \multicolumn{2}{|c|}{ All cases } \\
\hline & $\mathrm{n}$ & $\%$ & $\mathrm{n}$ & $\%$ & $\mathrm{n}$ & $\%$ \\
\hline \multicolumn{7}{|l|}{ Clinical extent of disease } \\
\hline Localised & 1060 & 36.5 & 192 & 20.1 & 1252 & 32.5 \\
\hline Regional & 791 & 27.3 & 185 & 19.4 & 976 & 25.3 \\
\hline Distant & 790 & 27.2 & 414 & 43.4 & 1204 & 31.2 \\
\hline Not known & 260 & 9.0 & 163 & 17.1 & 423 & 11.0 \\
\hline \multicolumn{7}{|l|}{ Tumour morphology } \\
\hline Squamous cell carcinoma & 938 & 40.7 & 168 & 30.6 & 1106 & 38.7 \\
\hline Small cell lung carcinoma & 532 & 23.1 & 146 & 26.6 & 678 & 23.7 \\
\hline Adenocarcinoma & 431 & 18.7 & 126 & 23.0 & 557 & 19.5 \\
\hline Other & 332 & 14.4 & 76 & 13.8 & 408 & 14.3 \\
\hline Not recorded/not known & 74 & 3.2 & 33 & 6.0 & 107 & 3.7 \\
\hline
\end{tabular}

\#: 999 cases (594 of RP cases, 20.5\% and 405 non-RP cases 42.5\%) without microscopic verification were excluded. 


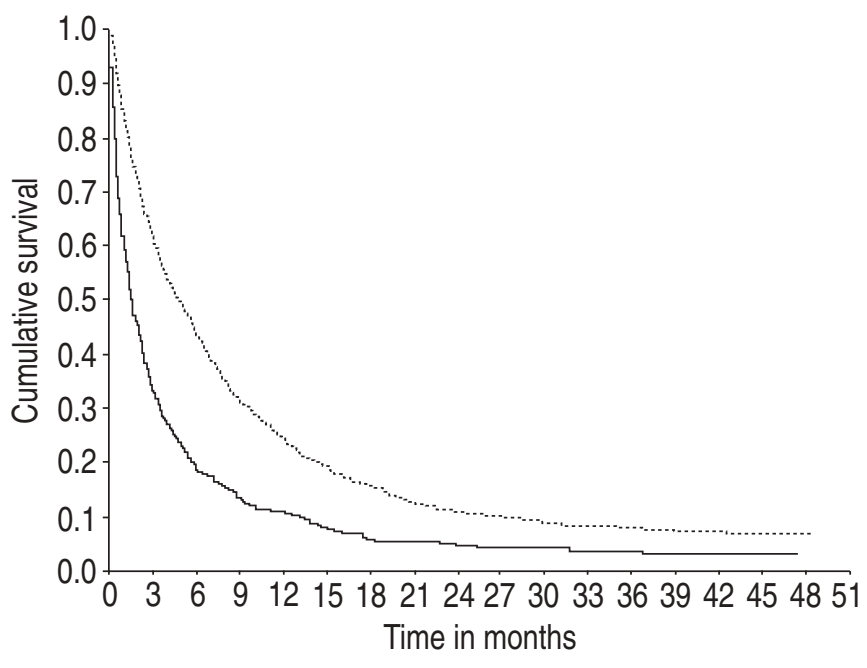

Fig. 2.-Kaplan-Meier survival curves for patients managed by a respiratory physician (RP: - ) or not managed by an RP within the first 6 months $(--) . \mathrm{p}<0.001$.

\section{Survival}

For all patients, the overall Kaplan-Meier estimates of survival at 1 and 3 yrs was $21.1 \%$ (95\% confidence interval (CI): $19.8-22.4 \%)$ and 7.0\% (95\% CI: 6.2-7.8\%), respectively. Patients managed by an RP had a better observed survival than patients with no RP involvement (fig. 2). The 1 and $3 \mathrm{yr}$ survival estimates for the RP patients were $24.4 \%$ $(22.8-26.0 \%)$ and $8.1 \%(7.1-9.1 \%)$, respectively. The corresponding figures for the patients with no RP involvement were $11.1 \%(9.1-13.1 \%)$ and $3.7 \%(2.5-4.8 \%)$. The log-rank test of equality of the two curves shown in figure 2 was highly significant $(\mathrm{p}<0.001)$. These observed differences in survival equate to a higher unadjusted hazard ratio of death of 1.81 (95\%CI: 1.67-1.95) for the non-RP patients versus the RP patients. After adjustment for the demographic factors (age, sex, deprivation and settlement size), the hazard ratio was reduced to $1.70(1.58-1.84)$ in a multivariate main effects Cox proportional-hazards model. With further adjustment for clinical extent of disease and morphology of tumour, the hazard ratio decreased to 1.44 (1.33-1.56). Finally, the addition of any active treatment as a further explanatory variable changed the hazard ratio to 1.41 (1.30-1.52), however, this still represents a significantly higher risk of death among patients not managed by an RP. Adjustment for management by an oncologist or a cardiothoracic surgeon did not further reduce this adjusted hazard ratio.

\section{Discussion}

Lung cancer management guidelines [2-4] assume that patients with the condition present in a typical fashion and follow a well set journey through primary and secondary care. Typically, new respiratory symptoms lead to the GP finding an abnormal radiograph and referring to a chest physician. Clinical experience indicates that this is far less typical than is generally believed. A recent report from the Yorkshire Cancer Registry outlined the referral pathways in secondary care in an unselected group of 400 patients [1]. Only $52 \%$ of patients were initially referred to a lung cancer specialist (chest physician, cardiothoracic surgeon or oncologist). There were 18 different specialities involved in the initial consultation and 70 unique referral pathways resulted. The purpose of this present study was to investigate the impact of the involvement of an RP on the investigation, treatment and survival of lung cancer patients. The data was taken from a wider study of lung cancer management in Scotland, which has been published elsewhere [6].

The study was population based with cases identified from a national cancer registry with a history of high levels of data accuracy [10] and case ascertainment [11]. This allowed capture of information on patients who were managed in primary care or in specialties that rarely deal with lung cancer patients. Since the majority of patients with this condition survive less than a year the authors thought it reasonable to investigate the effect of seeing a respiratory physician in the first 6 months only. The study was limited by the information having been extracted retrospectively from hospital and primary-care records and therefore, did not allow the collection of some known prognostic factors such as performance status, weight loss or the existence of significant comorbidity.

Patients managed by respiratory physicians were significantly younger than those who were seen by other clinicians, many of whom were geriatricians. This effect has previously been described by a group from Southend [12] who showed that age was a major factor in influencing treatment choices and referral patterns in an unselected group of 563 lung cancer patients presenting to a single health district, $85 \%$ of whom were managed by a chest physician. The data from this study further supports the existence of an age effect but also shows that in terms of prediction of survival, it is relatively weak.

The authors have shown that patients resident in rural areas were more likely to be managed by a respiratory physician than those who lived in more densely urban areas. This may offer some explanation for the conclusion of another recently published study based on Scottish Cancer Registry data [13] which showed that residency in a small settlement does not give a survival disadvantage in lung cancer. This presumably reflects the fact that respiratory physicians are well represented in all Scottish District General Hospitals and access to their services does not appear to be a problem.

Patients seeing respiratory physicians were more likely to have the stage of their disease recorded. These patients were also more likely to undergo more rigorous staging investigations such as CT scans and bronchoscopy. Some of this effect may be explained, however, by the fact that more patients seeing nonrespiratory physicians had metastatic disease which would presumably require less in the way of thoracic staging investigations. This is supported by the fact that tests assessing distant spread (liver ultrasound and CT scan of the brain) were more commonly performed in the non-RP group.

The microscopic confirmation rate was much higher in patients seen by a chest physician, a fact that has been reported elsewhere $[1,5,12]$. Other studies have concluded that lower histological confirmation rates, correlate with the delivery of less active treatment $[5,12,14]$ this study's data suggests that this may have an important influence on survival. Patients seeing respiratory physicians were also more likely to see other lung cancer clinicians such as oncologists and thoracic surgeons. This present study also shows that this translates into the delivery of more active treatment. This was especially true for treatments which are known to prolong life in lung cancer cases such as surgery, chemotherapy and radical radiotherapy.

Survival for all patients was poor, but those managed by $\mathrm{RP}$ appeared to fair better than those in the non-RP group. Clearly factors such as age, stage of disease and the delivery of active treatment had an influence on survival but even allowing for these, seeing an RP still held an overall survival advantage. The reasons for this are not clear and may be related to case mix factors such as comorbidity, poor 
performance status and weight loss which were not collected in the study. Also the quality of the staging data in each group may have influenced survival. The survival data from this study is similar to the Yorkshire Registry figures [14], which showed that the relative risk of death was significantly reduced by "specialist" management.

It is possible that patients seen by an RP had less delay in diagnosis and this may have an apparent effect on survival by creating lead-time bias. However, patients who had negative or no histology were allocated on an earlier date of diagnosis, by the authors definition, and these patients were more commonly seen in the non-RP group. Improved survival in the RP group may have resulted because of fewer delays in referral for treatments. However, this was not specifically measured in this present study and should be assessed in future prospective audits of lung cancer management. This may prove difficult as at present the only way of tracing unselected lung cancer patients is to use registry data which makes prospective audit impossible.

The data from this study supports the published national guidelines that lung cancer patients benefit from the involvement of a respiratory physician [3]. In general these patients are younger and have a more favourable stage of disease; however, these factors alone do not explain the survival advantage of seeing a respiratory physician. Patients seeing a respiratory physician are more likely to be managed in a multidisciplinary way and receive more active treatment. Further studies are required to investigate the reasons for the differences seen in this study. If possible they should also investigate whether referral to a respiratory physician and the multidisciplinary lung cancer team is associated with a better quality of survival. Clearly the challenge in the future is to improve the access of all lung cancer patients to the multidisciplinary lung cancer team.

Acknowledgements. The authors would like to thank members of the former Scottish Lung Cancer Focus Group for their help with the design of the study; the Scottish Cancer Therapy Network(SCTN) Data Managers for their help with data validation and support; and members of the Scottish Cancer Trials Lung Group (SCTLG) for their comments on the text.

Members of the Scottish Cancer Trials Lung Group (SCTLG). R. Milroy, D.H. Brewster, P. Brown, D.J. Dunlop, M Fallon, R.J. Fergusson,
A. Gregor, R. Jones, K.M. Kerr, A.J. Kirk, J.B. MacDonald, J. McPhelim, M. Nicolson, N. O'Rourke, A. Price, E. Rankin, M. Storrie, A. White.

\section{References}

1. Northern and Yorkshire Cancer Registry and Information Service (NYCRIS). Lung Cancer Referral Patterns. The Yorkshire Experience. Leeds, NYCRIS, 2000.

2. The Lung Cancer Working Party of the British Thoracic Society Standards of Care Committee. BTS recommendations to respiratory physicians for organising the care of patients with lung cancer. Thorax 1998; 53: Suppl. 1, S1-S8.

3. Scottish Intercollegiate Guideline Network. No 23, Management of Lung Cancer. Edinburgh, SIGN, 1998.

4. Royal College of Radiologists' Clinical Oncology Information Network. Guidelines on the non-surgical management of lung cancer. Clin Oncol 1999; 11: S1-S53.

5. Muers MF, Haward RA. Management of lung cancer. Thorax 1996; 51: 557-560.

6. Gregor A, Thomson CS, Brewster DH, et al. Management and survival of lung cancer patients diagnosed in 1995 in Scotland: results of a national, population-based study. Thorax 2001; 56: 212-217.

7. Carstairs V, Morris R. Deprivation and Health in Scotland. Aberdeen, Aberdeen University Press, 1991.

8. Kendrick S, Clarke J. The Scottish Record Linkage System. Health Bulletin 1993; 51: 72-79.

9. Collett D. Modelling Survival Data in Medical Research. London, Chapman and Hall, 1994.

10. Brewster D, Crichton J, Muir C. How accurate are Scottish cancer registration data? Br J Cancer 1994; 70: 954-959.

11. Brewster D, Crichton J, Harvey JC, Dawson G. Completeness of case ascertainment in a Scottish Regional Cancer Registry for the year 1992. Public Health 1997; 111: 339-343.

12. Brown JS, Eraut D, Trask C, Davison AG. Age and the treatment of lung cancer. Thorax 1996; 51: 564-568.

13. Campbell NC, Elliott AM, Sharp L, Ritchie LD, Cassidy J, Little J. Rural factors and survival from cancer: analysis of Scottish cancer registrations. Br J Cancer 2000; 82: 18631866.

14. Northern and Yorkshire Cancer Registry and Information Service (NYCRIS). Cancer treatment policies and their effects on survival. Key sites study 2. Lung. Leeds, NYCRIS, 1999. 